\title{
Inflation as a White Hole explosion from a 5D vacuum
}

\author{
1,2 Mariano Anabitarte*, and ${ }^{1,2}$ Mauricio Bellini ${ }^{\dagger}$ \\ 1 Departamento de Física, \\ Facultad de Ciencias Exactas y Naturales, \\ Universidad Nacional de Mar del Plata, \\ Funes 3350, (7600) Mar del Plata, Argentina. \\ 2 Instituto de Investigaciones Físicas de Mar del Plata (IFIMAR), \\ Consejo Nacional de Investigaciones Científicas y Técnicas (CONICET), Argentina.
}

\begin{abstract}
Using a new kind of 5D Ricci-flat canonical metric, we obtain by a static foliation an effective 4D Schwarzschild-de Sitter hypersurface. We examine some particular initial conditions which could be responsible for the inflationary expansion of the early universe, which could be driven by the explosion of a White Hole (WH). The zeroth order spectrum outside the WH describes quantum fluctuations, which for a scale invariant power spectrum, can be expressed in terms of the cosmological constant, or the square mass of the WH.
\end{abstract}

\footnotetext{
* anabitar@mdp.edu.ar

† mbellini@mdp.edu.ar
} 


\section{MOTIVATION}

The Induced Matter Theory (IMT) [1] is based on the assumption that ordinary matter and physical fields that we can observe in our 4D universe can be geometrically induced from a 5D Ricci-flat metric with a space-like noncompact extra dimension on which we define a physical vacuum. The Campbell-Magaard Theorem (CMT)[2] serves as a ladder to go between manifolds whose dimensionality differs by one. Due to this theorem one can say that every solution of the 4D Einstein equations with arbitrary energy momentum tensor can be embedded, at least locally, in a solution of the 5D Einstein field equations in a relativistic vacuum: $G_{A B}=0$. For this reason the stress-energy may be a $4 \mathrm{D}$ manifestation of the embedding geometry and therefore, by making a static foliation on the space-like extra coordinate of an extended 5D de Sitter spacetime, it is possible to obtain an effective $4 \mathrm{D}$ universe that suffered an exponential accelerated expansion driven by an effective scalar field with an equation of state typically dominated by vacuum[4-7]. In this work we shall consider a new kind of 5D Ricci-flat static canonical metric

$$
d S_{(5)}^{2}=F^{2}(\psi)\left[f(r) d t^{2}-\frac{d r^{2}}{f(r)}-r^{2}\left(d \theta^{2}+\sin ^{2}(\theta) d \phi^{2}\right)\right]-\frac{3}{\Lambda}\left(\frac{d F(\psi)}{d \psi}\right)^{2} d \psi^{2}
$$

Here, $F(\psi)$ is a dimensionless function of the extra coordinate, $f(r)=1-\frac{2 G m}{r}-\frac{\Lambda r^{2}}{3}$ is a dimensionless function, $\psi$ is the space-like and non-compact fifth extra coordinate ${ }^{1}$. This is a generalization of the group of 5D Ricci-flat canonical metrics, that extends a 4D Swarzschild - de Sitter (SdS) metric. The coordinate $t$ is time-like and $r, \theta, \phi$ are the usual spherical polar coordinates. Furthermore, $\Lambda$ is the cosmological constant, $m$ is the mass of the gravitational source and we shall consider that the speed of light is dimensionless: $c=1$. The metric (1) is very interesting because avoids naked singularities for $m<\frac{1}{3 G \sqrt{\Lambda}}$. There cannot be sources with masses $m>\frac{1}{3 G \sqrt{\Lambda}}$, because in these cases all the roots are complex.

In this work we shall consider the particular case where the mass takes the particular value $m \equiv \frac{\psi_{0}}{3 \sqrt{3} G}=\frac{1}{3 G \sqrt{\Lambda}}$. If we foliate $\psi \equiv \psi_{0}=\sqrt{\frac{3}{\Lambda}}$, the resulting $4 \mathrm{D}$ hypersurface describes a SdS static spacetime with particular physical properties. The resulting effective $4 \mathrm{D}$ hypersurface will be a spacetime which has a scalar curvature ${ }^{(4)} R=\frac{\epsilon}{4 \Phi^{2}}\left[g^{\mu \nu}{ }_{4} g_{\mu \nu, 4}+\left(g^{\mu \nu} g_{\mu \nu, 4}\right)^{2}\right]$ [see [8] and references therein], such that in our case $\epsilon=-1$ and $\Phi^{2}=\frac{\Lambda}{12(d F / d \psi)^{2}}$. After making the

\footnotetext{
${ }^{1}$ In our notation conventions henceforth, indices $a, b=$ run from 0 to 4 , whereas the rest of indices $i, j, n, l, \ldots=$ run from 1 to 3 .
} 
foliation, we obtain the effective well known 4D SdS metric

$$
d S_{(4)}^{2}=\left[f(r) d t^{2}-\frac{d r^{2}}{f(r)}-r^{2}\left(d \theta^{2}+\sin ^{2}(\theta) d \phi^{2}\right)\right] .
$$

For this particular mass value, the horizon is unique and takes the value ${ }^{2}: r_{*}=\frac{1}{\sqrt{\Lambda}}$. Furthermore, at $r=r_{*}$ the effective $4 \mathrm{D}$ components of the Ricci-tensor are zero: $\left.R_{\alpha \beta}\right|_{r_{*}}=0$, so that at this point the spacetime is locally flat. But is this a causal horizon? From the strict point of view at this point there is no causal horizon because while it is true that $f\left(r \equiv r_{*}\right)=0$, $f(r)$ has the same sign for any other value of $r \neq r_{*}: f\left(r \lessgtr r_{*}\right)<0$. Moreover, the bilinear form $d S_{(4)}^{2}$ has the same sign on both sides of $r_{*}$. However, here arises a difference between the physical properties for $r<r_{*}$ and $r>r_{*}$. For $r<r_{*}$ the gravitational field (in absence of angular moment) is attractive, but for $r>r_{*}$ is repulsive. This means that outside the false horizon the gravitational field is always repulsive. Hence, observers who are at $r>r_{*}$ can see a white hole with a false horizon at $r=r_{*}$.

This can be seen from another point of view: for a massive test particle outside a spherically symmetric compact object in 5D with an exterior metric given by (1) the 5D Lagrangian can be written as $L_{(5)}=\frac{1}{2} g_{a b} U^{a} U^{b}$. As it is usually done in literature we can, without loss of generality, confine the test particles to describe orbits with $\theta=\pi / 2$. Thus, from the Lagrangian (11), it can be easily seen that only $t$ and $\phi$ are cyclic coordinates, so their associated constants of motion $p_{t}$ and $p_{\phi}$, are $p_{t} \equiv \frac{\partial L_{(5)}}{\partial U^{t}}$ and $p_{\phi} \equiv \frac{\partial L_{(5)}}{\partial U^{\phi}}$. Since we are dealing with the case where $f(r)<0$, the five-velocity condition for a time-like geodesic will be

$$
g_{a b} U^{a} U^{b}=-1
$$

One can use with the constants of motion $p_{t}$ and $p_{\phi}$, and considering $\theta=\pi / 2$, we obtain the equation for the energy conservation. After making a static foliation $\psi=\sqrt{\frac{3}{\Lambda}}$ [which implies that the observer is moving with $U^{\psi}=0$ and $F^{2}\left(\psi=\sqrt{\frac{3}{\Lambda}}\right)=1$ ], can be obtained the effective $4 \mathrm{D}$ equation of the energy for a test particle on the SdS spacetime: $\frac{1}{2}\left(U^{r}\right)^{2}+V_{\text {ind }}(r)=E_{\text {ind }}$, with

$$
\begin{aligned}
V_{\text {ind }}(r) & =-\frac{1}{2}\left\{\left[f(r)+\frac{\Lambda}{3} r^{2}\right] \frac{p_{\phi}^{2}}{r^{2}}-[f(r)-1]\right\}, \\
E_{\text {ind }} & =\frac{1}{2}\left[p_{t}^{2}+\frac{\Lambda}{3} p_{\phi}^{2}-1\right] .
\end{aligned}
$$

\footnotetext{
${ }^{2}$ There are three roots, but two of them are complex.
} 
The interesting thing is that, for $p_{\phi}=0$, (i.e., when the field is purely gravitational), the Newtonian acceleration $a_{N}=-d V_{\text {ind }} / d r$, is given by

$$
a_{N}=-\sqrt{\frac{\Lambda}{3}} \frac{1}{r^{2}}+\frac{\Lambda}{3} r
$$

and this acceleration is negative for $r<r_{*}$, but positive for $r>r_{*}$, so that for sufficiently large scales the effective gravitational field becomes repulsive. In the following we shall explore the cosmological consequences of repulsive gravity in the early universe.

\section{INFLATION FROM A WH EXPLOSION}

As we can see, the metric (1) is written on a static chart coordinate. In order to get it written on a dynamical chart coordinate $\{t, r, \theta, \phi\}$, let us use the coordinate transformation[ 9$]$ given by

$$
R=\operatorname{ar}\left[1+\frac{1}{6 a r \sqrt{\Lambda}}\right]^{2}, \quad T=t+\sqrt{\frac{\Lambda}{3}} \int^{r} d R \frac{R}{f(R)}\left(1-\frac{2}{3 \sqrt{\Lambda} R}\right)^{-1 / 2}, \psi=\psi,
$$

where $a(t)=e^{\sqrt{\frac{1}{3}} t}$ is the scale factor, and $\sqrt{\frac{\Lambda}{3}}$ is the Hubble constant. Thus the line element (1) can be written in terms of the conformal time $\tau$ as

$$
d S_{(5)}^{2}=[F(\psi)]^{2}\left[U(\tau, r) d \tau^{2}-V(\tau, r)\left(d r^{2}+r^{2}\left(d \theta^{2}+\sin ^{2} \theta d \phi^{2}\right)\right)\right]-\frac{3}{\Lambda}\left(\frac{d F(\psi)}{d \psi}\right)^{2} d \psi^{2},
$$

where the metric functions $U(\tau, r)$ and $V(\tau, r)$ are given by

$$
U(\tau, r)=a^{2}(\tau)\left[1-\frac{1}{6 a r \sqrt{\Lambda}}\right]^{2}\left[1+\frac{1}{6 a r \sqrt{\Lambda}}\right]^{-2}, \quad V(\tau, r)=a^{2}(\tau)\left[1+\frac{1}{6 a r \sqrt{\Lambda}}\right]^{4},
$$

with $d \tau=a^{-1}(\tau) d t$ and $a(\tau)=-1 /\left(\sqrt{\frac{\Lambda}{3}} \tau\right)$, so that the Hubble parameter is a constant given by $\sqrt{\frac{\Lambda}{3}}=a^{-2} \frac{d a}{d \tau}$.

Now we consider a 5D massless scalar field which is free of any interactions. The dynamics is given by a Klein-Gordon equation: $\frac{1}{\sqrt{\left|g_{(5)}\right|}} \frac{\partial}{\partial y^{a}}\left[\sqrt{\left|g_{(5)}\right|} g^{a b} \varphi_{, b}\right]=0$, where $\sqrt{\left|g_{(5)}\right|}=F^{4}(\psi) \sqrt{\frac{3}{\Lambda}}|d F / d \psi| U^{1 / 2} V^{3 / 2} r^{2} \sin \theta$ is the determinant of the covariant metric tensor $g_{a b}$. It can be demonstrated that $\varphi(\tau, r, \theta, \phi, \psi)$ can be separated in the form $\varphi(\tau, r, \theta, \phi, \psi) \sim \Phi(\tau, r) G(\theta, \phi) \Omega(\psi)$, such that the differential equation for $\Omega(\psi)$ is

$$
\Omega^{\prime \prime}+\left[\frac{4(\Lambda / 3)^{2}}{\left(F^{\prime}\right)^{3} F}-\frac{F^{\prime \prime}}{F^{\prime}}\right] \Omega^{\prime}-\left(\frac{\Lambda M^{2}}{3\left(f^{\prime}\right)^{2} f^{2}}\right) \Omega(\psi)=0,
$$

where $M^{2}$ is a separation constant and the prime denotes the derivative with respect to $\psi$. 


\section{A. The 4D induced field equation}

Assuming that the 5D spacetime can be foliated by the hypersurface $\psi=\sqrt{\frac{3}{\Lambda}}$, from the metric (8) we obtain that the $4 \mathrm{D}$ induced metric is given by

$$
d S_{(4)}^{2}=U(\tau, r) d \tau^{2}-V(\tau, r)\left[d r^{2}+r^{2}\left(d \theta^{2}+\sin ^{2} \theta d \phi^{2}\right)\right]
$$

where the metric functions $U(\tau, r)$ and $V(\tau, r)$ are given by (9). We shall consider that $\bar{\varphi}(\tau, r, \theta, \phi)=\varphi\left(\tau, r, \theta, \phi, \psi_{0}\right)$ is the effective scalar field induced on the effective 4D hypersurface obtained after making the foliation. Furthermore, for consistency we shall require that $F\left(\psi=\sqrt{\frac{3}{\Lambda}}\right)=1$, in order to make $\left.\sqrt{\left|g_{(5)}\right|}\right|_{\psi=\sqrt{\frac{3}{\Lambda}}}=\sqrt{\left|g_{(4)}\right|}$, where $g_{(4)}$ is the determinant of the metric (11). We expand the induced scalar field $\bar{\varphi}$ as [10]

$$
\bar{\varphi}(\vec{r}, \tau)=\int_{0}^{\infty} d k \sum_{l m}\left[a_{k l m} \bar{\Phi}_{k l m}(\vec{r}, \tau)+a_{k l m}^{\dagger} \bar{\Phi}_{k l m}^{*}(\vec{r}, \tau)\right],
$$

where $\bar{\Phi}_{k l m}(\vec{r}, \tau)=k^{2} j_{l}(k r) \bar{\Phi}_{k l}(\tau) Y_{l m}(\theta, \phi)$, such that $Y_{l m}(\theta, \phi)$ are the spherical harmonics, $j_{l}(k r)$ are the spherical Bessel functions and the annihilation and creation operators obey the algebra $\left[a_{k l m}, a_{k^{\prime} l^{\prime} m^{\prime}}^{\dagger}\right]=\delta\left(k-k^{\prime}\right) \delta_{l l^{\prime}} \delta_{m m^{\prime}},\left[a_{k l m}, a_{k^{\prime} l^{\prime} m^{\prime}}\right]=\left[a_{k l m}^{\dagger}, a_{k^{\prime} l^{\prime} m^{\prime}}^{\dagger}\right]=0$. The mean square fluctuations can be obtained by using the theorem of the spherical harmonics

$$
\left\langle E\left|\bar{\varphi}^{2}(\vec{r}, \tau)\right| E\right\rangle=\int_{0}^{\infty} \frac{d k}{k} \sum_{l} \frac{2 l+1}{4 \pi} k^{5} j_{l}^{2}(k r)\left|\bar{\Phi}_{k l}(\tau)\right|^{2},
$$

where $|E\rangle$ is an arbitrary quantum state. We shall assume that ${ }^{3} \bar{\varphi}(\tau, r, \theta, \phi)=\bar{\Phi}(\tau, r) \bar{G}(\theta, \phi)$. Next,using the fact that $\epsilon$ is a small we propose the following expansion for $\bar{\Phi}_{l}$ in orders of $\epsilon$ : $\bar{\Phi}_{l}(r, \tau)=\bar{\Phi}_{l}^{(0)}+\bar{\Phi}_{l}^{(1)}+\ldots$. Thus, we are now able to calculate solutions for $\bar{\Phi}_{l}(r, \tau)$ at zeroth order in the expansion. The spectrum for the square fluctuations (13) can be calculated at zeroth order

$$
\mathcal{P}_{k}(\tau)=\frac{k^{3}}{2 \pi^{2}}\left|\bar{\Phi}_{k l}^{(0)}\right|^{2}+\ldots
$$

\footnotetext{
${ }^{3}$ The equation for $\bar{\Phi}(r, \tau)$ on the effective 4D hypersurface described by (11), and can be written as

$$
\frac{\partial^{2} \bar{\Phi}_{l}}{\partial \tau^{2}}-\frac{2}{\tau} \frac{\partial \bar{\Phi}_{l}}{\partial \tau}-\frac{2}{r} \frac{\partial \bar{\Phi}_{l}}{\partial r}-\frac{\partial^{2} \bar{\Phi}_{l}}{\partial r^{2}}-\left[\frac{l(l+1)}{r^{2}}-M^{2}\right] \bar{\Phi}_{l}
$$$$
=\left(1-\frac{V}{U}\right) \frac{\partial^{2} \bar{\Phi}_{l}}{\partial \tau^{2}}-\left[\frac{2}{\tau}+\frac{1}{\sqrt{U V}} \frac{\partial}{\partial \tau}\left(\frac{V^{3}}{U}\right)^{1 / 2}\right] \frac{\partial \bar{\Phi}_{l}}{\partial \tau}-M^{2}(V-1) \bar{\Phi}_{l}+\frac{1}{2}\left(\frac{1}{U} \frac{\partial U}{\partial r}+\frac{1}{V} \frac{\partial V}{\partial r}\right) \frac{\partial \bar{\Phi}_{l}}{\partial r} .
$$ 


\section{B. Scalar field dynamics and zeroth order power spectrum outside the WH}

The dynamics for a massive scalar field in a spatially homogeneous de Sitter case is described by

$$
\frac{\partial^{2} \bar{\Phi}_{l}^{(0)}}{\partial \tau^{2}}-\frac{2}{\tau} \frac{\partial \bar{\Phi}_{l}^{(0)}}{\partial \tau}-\frac{2}{r} \frac{\partial \bar{\Phi}_{l}^{(0)}}{\partial r}-\frac{\partial^{2} \bar{\Phi}_{l}^{(0)}}{\partial r^{2}}-\left[\frac{l(l+1)}{r^{2}}-M^{2} a^{2}(\tau)\right] \bar{\Phi}_{l}^{(0)}=0,
$$

where the last term corresponds to the induced mass of the scalar field. If we use the Bessel transform: $\bar{\Phi}_{l}^{(0)}(r, \tau)=\int_{0}^{\infty} d k k^{2} j_{l}(k r) \bar{\Phi}_{k l}^{(0)}(\tau)$, we obtain the zeroth order dynamics for the $\operatorname{modes} \bar{\Phi}_{k l}^{(0)}$

$$
\frac{\partial^{2} \bar{\Phi}_{k l}^{(0)}}{\partial \tau^{2}}-\frac{2}{\tau} \frac{\partial \bar{\Phi}_{k l}^{(0)}}{\partial \tau}+\left(k^{2}+\frac{3 M^{2}}{\Lambda \tau^{2}}\right) \bar{\Phi}_{k l}^{(0)}=0
$$

If we use the Bunch-Davies vacuum, we obtain the normalized modes solution: $\bar{\Phi}_{k l}^{(0)}=$ $A_{1}(-\tau)^{3 / 2} \mathcal{H}_{\nu}^{(1)}[-k \tau]+A_{2}(-\tau)^{3 / 2} \mathcal{H}_{\nu}^{(2)}[-k \tau]$, where $\mathcal{H}_{\nu}^{(1,2)}$ are respectively the first and second kind Hankel functions with $\nu^{2}=\frac{9}{4}-\frac{M^{2}}{H^{2}}$. The normalization constants are $A_{2}=$ $-\frac{\sqrt{\pi} \sqrt{\Lambda}}{2 \sqrt{3}} e^{-i \nu \pi / 2}, A_{1}=0$. We are interested in the spectrum of the fluctuations without sources, such that $l=0$. The spectrum is

$$
\mathcal{P}_{k l}^{(0)}(\tau)=\frac{k^{3}}{2 \pi^{2}}\left|\bar{\Phi}_{k l}^{(0)}\right|^{2}=\frac{\Lambda}{3 \pi}\left(\frac{-k \tau}{2}\right)^{3} \mathcal{H}_{\nu}^{(2)}[-k \tau] \mathcal{H}_{\nu}^{(1)}[-k \tau]
$$

where $k$ given by $k=\frac{2 \pi}{a(\tau) r}\left[1+\frac{1}{2 \sqrt{3} \sqrt{\Lambda} a(\tau) r}\right]^{-2}$, is the wave number on a physical frame.

Outside the WH the fluctuations are small and the wavenumber of these fluctuations on scales of size $\frac{\sqrt{3}}{\sqrt{\Lambda}}$, is

$$
k_{H} \simeq \frac{2 \pi}{a(\tau) r_{H}}=-\frac{2 \pi \sqrt{\Lambda} \tau}{\sqrt{3} r_{H}},
$$

where we have made use of the fact that $a(\tau)=-\sqrt{3} /(\sqrt{\Lambda} \tau)$, with $\tau \leq 0$. Furthermore, at the end of inflation $\tau \rightarrow 0$, so that $\mathcal{H}_{\nu}^{(2)}[-k \tau] \simeq \frac{-i}{\pi} \Gamma(\nu)(-k \tau / 2)^{-\nu}$. The power spectrum on scales close to the Hubble horizon is

$$
\left.\mathcal{P}_{k l}^{(0)}(\tau)\right|_{H}=\frac{k^{3}}{2 \pi^{2}}\left|\bar{\Phi}_{k l}^{(0)}\right|_{H}^{2} \simeq\left(\frac{\pi \sqrt{\Lambda} \tau^{2}}{\sqrt{3} r_{H}}\right)^{3-2 \nu} \frac{\Gamma^{2}(\nu) \Lambda}{3 \pi^{3}}
$$

which depends on the mass of the inflaton field $M$, because $\nu^{2}=\frac{9}{4}-\frac{3 M^{2}}{\Lambda}$. For a nearly scale invariant power spectrum: $\nu \simeq 3 / 2$, we obtain

$$
\left.\mathcal{P}_{k l}^{(0)}(\tau)\right|_{H, \nu \simeq 3 / 2} \simeq \frac{\Lambda}{12 \pi^{2}}
$$

which describe quantum fluctuations. Notice the dependence of this spectrum with the cosmological constant (or the square mass of the $\mathrm{WH}$ ). 


\section{FINAL COMMENTS}

Using a new kind of canonical metric here proposed, we have explored the possibility that the inflationary early universe could be driven by the explosion of a WH with a mass which is related to the value of the foliation of the non-compact extra dimension $\left[\psi=\psi_{0}\right]$,

and hence of the cosmological constant: $m \equiv \frac{\psi_{0}}{3 \sqrt{3} G}=\frac{1}{3 G \sqrt{\Lambda}}$. This idea appears to be very attractive because excludes the problem of the initial singularity taking into account the idea of a large-scale repulsive gravitational field.

\section{Acknowledgements}

The authors acknowledge UNMdP and CONICET Argentina for financial support.

[1] P. S. Wesson, Phys. Lett. B276: 299 (1992);

J. M. Overduin and P. S. Wesson, Phys. Rept. 283: 303 (1997).

[2] J. E. Campbell, A course of Differential Geometry (Clarendon, Oxford, 1926).

[3] L. Magaard, Zur einbettung riemannscher Raume in Einstein-Raume und konformeuclidische Raume. (PhD Thesis, Kiel, 1963).

[4] A. Feinstein. Phys. Rev. D66: 063511 (2002).

[5] M. Sami. Mod. Phys. Lett. A18: 691 (2003).

[6] D. S. Ledesma, M. Bellini. Phys. Lett. B581: 1 (2004).

[7] M. Bellini. Phys. Lett. 609: 208 (2005).

[8] Paul S. Wesson. Classical and Quantum Consequences of Kaluza-Klein Cosmology. World Scientific Publishing. Singapure (2006).

[9] T. Shiromizu, D. Ida and T, Torii, J. High Energy Physics 11: 010 (2001).

[10] J. E. Madriz Aguilar, M. Bellini, JCAP 1011: 020(2010). 